\title{
DIFFERENTIAL SCANNING CALORIMETRY ON MIXTURES OF LECITHIN, LYSOLECITHIN AND CHOLESTEROL
}

\author{
W.E. KLOPFENSTEIN *, B. de KRUYFF, A.J. VERKLEIJ, R.A. DEMEL and \\ L.L.M. van DEENEN \\ Laboratory of Biochemistry, University of Utrecht, University Centre 'De Uithof", Padualaan 8, \\ Utrecht, The Netherlands
}

Received March 7, 1974, accepted June 25, 1974

The effect of increasing concentrations of lysolecithin (1-palmitoyl-sn-glycerol-3-phosphorylcholine) on the gel $\rightarrow$ liquid crystal thermal transition of lecithin (1,2-dipalmitoyl-sn-glycerol3-phosphorylcholine) in the aque ous phase was studied by differential scanning calorimetry (DSC). Ly solecithin showed an endothermic transition at $3.4^{\circ} \mathrm{C}$ whereas the transition of the lecithin occurred at $42^{\circ} \mathrm{C}$. No phase separation could be observed calorimetrically at lysolecithin concentrations up to $60 \mathrm{~mol} \%$. Freeze etch electron microscopy showed that mixtures containing as much as $50 \mathrm{~mol} \%$ lysolecithin exist in a lamellar phase. The lysolecithin was found to cause an initial slight increase in the enthalpy of transition followed by a gradual decrease. The enthalpy increased again at very high lysolecithin concentrations. The lysolecithin also caused a non-linear decrease in the temperature at which the lecithin transition took place.

Cholesterol was found to decrease the enthalpy of transition of the lysolecithin, eliminating it at a concentration of $50 \mathrm{~mol} \%$. Cholesterol caused an increase in the temperature at which the lysolecithin transition took place.

\section{Introduction}

Lysolecithin has long been of interest because of its ability to interact with and disrupt membranes. However, the mechanism of lysolecithin's interaction with the membrane and the subsequent disruption is not understood. To gain additional information on this process there have been studies on lysis by lysolecithin analogs, synthetic lysolecithins of various hydrocarbon chain lengths, lytic lecithins [1] and modified lysolecithins [2]. The mere presence of lysolecithin in a membrane is not sufficient to cause lysis. Ibrahim and Thompson [3] showed that hydrolysis of nearly $20 \%$ of the phospholipids of intact erythrocytes by sea snake phospholipase A caused negligible lysis. Van Zutphen and van Deenen [4] found that stable black lipid membranes can be formed from egg lecithin containing up to $15 \%$ lysolecithin but not with $20 \%$ lysolecithin. The electrical resistance, however, was reduced

* Present address: Department of Biochemistry, Kansas State University, Manhattan, Kansas 66506, USA. 
100 -fold by the presence of $2 \%$ lysolecithin in the membrane. Reman [5] found by $\mathrm{X}$-ray diffraction that lysolecithin in egg lecithin up to concentrations of 40 mole\% caused the lipid leaflet to thin. At higher lysolecithin concentrations a phase separation was apparent. On the other hand, a lytic lecithin (didecanoyl lecithin) up to concentrations of more than 70 mole $\%$, caused a continued thinning of the leaflet with no apparent phase separation.

In this study the effect of a lysolecithin of known structure on the thermal transition of a lecithin of known structure, and the effect cholesterol on the thermal transition of lysolecithin were investigated.

\section{Materials and methods}

Dipalmitoyl phosphatidylcholine was synthesized by standard procedures [6]. The product contained more than $99 \%$ palmitic acid by gas-liquid chroma sgraphy and was approximately $99 \%$ phosphatidylcholine as determined by thin-layur chromatography. Lysolecithin was prepared by phospholipase A degradation of dipalmitoyl lecithin. Cholesterol, (cholest-5-en-3 $\beta$-ol) was purchased from Fluka AG (Buchs, Switzerland).

The calorimetry was performed on a Perkin-Elmer DSC-1B instrument at range 1 unless otherwise noted. The usual heating rate was $8^{\circ} \mathrm{C} / \mathrm{min}$. The instrument was calibrated with distilled water, cyclohexane and naphthalene. An empty gold pan was used in the reference cell.

Liposomal lipid suspensions were prepared in distilled water or $50 \%$ ethylene glycol-water by agitation on a Vortex mixer while heating the mixture above the transition temperature of the lipid.

Quantities of lipids contained in the sample cells were determined by phosphorus analysis using the method of Fiske and Subbarow [7].

The samples for freeze-etch electron microscopy were quenched from $0^{\circ} \mathrm{C}$ and treated further as described by Ververgaert et al. [8,9].

\section{Results and discussion}

The gel $\rightarrow$ liquid crystal transition observed for dipalmitoyl lecithin took place at $42.0^{\circ} \mathrm{C}$ and was quite sharp. This value falls within the range of $38^{\circ} \mathrm{C}$ reported by Lippert and Peticolas [10] as determined by laser Raman and $43.2^{\circ} \mathrm{C}$ reported by Giannoni et al. [11] as determined by DSC, but is $1^{\circ} \mathrm{C}$ above the generally accepted transition temperature of $41.0^{\circ} \mathrm{C}[12,13]$. No pre-transition was observed in the heat curve probably due to the presence of $50 \%$ ethylene glycol. Sample sizes and heating rates were kept constant so that the experiments were internally consistent. The heat of the gel $\rightarrow$ liquid crystal transition observed was $9.2 \pm 0.4$ $\mathrm{kcal} / \mathrm{mol}$. This value is intermediate between the values reported previously $[12,13]$. 


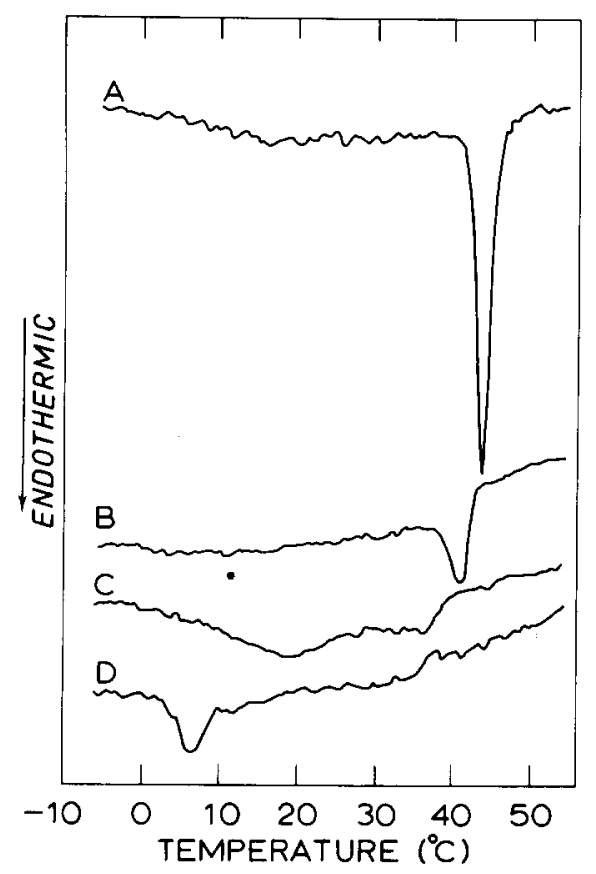

Fig. 1. Calorimetric scans showing the thermal transitions of lecithin-lysolecithin mixtures. Mixture A contained $10 \%$ lysolecithin; B, $61 \%$ lysolecithin; C, $89 \%$ lysolecithin, and D, $92 \%$ lysolecithin. Mixtures were prepared by mixing appropriate volumes of stock solutions of each component in a test tube, then drying them under a stream of $\mathrm{N}_{2}$, followed by vacuum drying overnight. The resultant residues were suspended in $50 \%$ ethylene glycol.

At $3.4^{\circ} \mathrm{C}$ the palmitoyl lysolecithin underwent a thermal transition, the heat of which was $4.5 \pm 0.8 \mathrm{kcal} / \mathrm{mol}$. It is of interest to note that although the "melting" heat per mole of lysolecithin is only half of that for the diacyl compound, the heat per fatty acid residue is essentially identical in the two compounds. Since the same heat is required to convert one fatty acid residue of either the monoacyl or diacyl compound from the gel to the liquid crystalline state, the chains must exist in similar environments, i.e. the packing of the fatty acids in the micellar structure of lysolecithin $[17,18]$ must be similar to that found in the lamellar structure of lecithin.

The effect of increasing the concentration of lysolecithin on the thermal transition of lecithin is shown in figs. 1 through 3 . The thermal transition of lecithin containing a small quantity of lysolecithin was quite sharp (fig. 1A). When the concentration of lysolecithin was increased to $61 \%$ (fig. 1B), the sharpness of the transition decreased markedly, accompanied by a decreased enthalpy of transition. A somewhat higher concentration of lysolecithin caused the appearance of a new broad transition at a temperature between those of lecithin and lysolecithin (fig. 1C). Because 


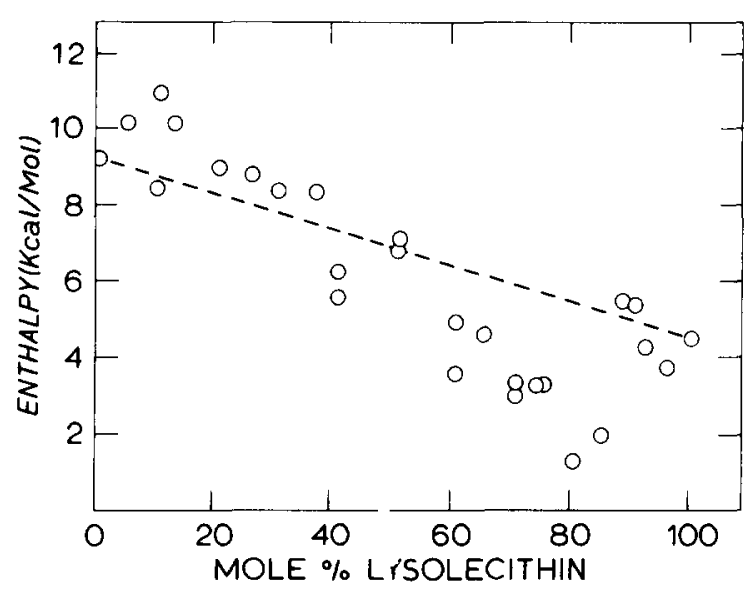

Fig. 2. Effect of lysolecithin on the enthalpy of transition of lecithin. The heat of transition for each reaction mix ture was calculated. This, together with the knowledge of the moles of phospholipid in the reaction cell, made it possible to calculate the enthalpy of transition per mole of phospholipid present. The circles represent the observed values; the broken line, the values predicated on the basis of $4.6 \mathrm{kcal} / \mathrm{mole}$ of hydrocarbon chain.

this transition was rather broad the starting temperature was difficult to determine, with the model 1B calorimeter. When this sample was on the more sensitive Perkin-Elmer DSC-2B it appeared that this intermediate transition began at $13^{\circ} \mathrm{C}$. The later transition which is the remnant of the lecithin transition peak, occurred at $30^{\circ} \mathrm{C}$.

A further increase in lysolecithin concentration (fig. 1D) caused the appearance of the lysolecithin transition peak, although a trace of a transition was still visible at about $30^{\circ} \mathrm{C}$ (confirmed by analysis on the model $2 \mathrm{~B}$ instrument). The intermediate peak was not pronounced but could have accounted for the tailing effect observed on the lysolecithin transition.

The effect of lysolecithin on the enthalpy of transition of lecithin is shown in fig. 2. The enthalpy of transition for the lecithin with two palmitic acid residues is $9.2 \mathrm{kcal}$ and that for lysolecithin is $4.5 \mathrm{kcal}$. Apparently, $4.5-4.6 \mathrm{kcal}$ is required to "melt" a palmitic acid residue in either of those compounds. The broken line in fig. 2 shows the enthalpies of transition expected on this basis for mixtures of the lecithin and lysolecithin. The observed values deviated from those expected. The slight initial rise in enthalpy values when lysolecithin was added was interpreted as an increased stability of the lecithin lamellar structure by the lysolecithin. Reman [5] observed that adding $10 \%$ lysolecithin to egg lecithin caused a marked thinning of the lipid leaflet and that further additions had less effect. He found that phase separation occurred above 40 mole $\%$ of lysolecithin, approximately the concentration at which the line for observed enthalpies in our study dropped below the line 


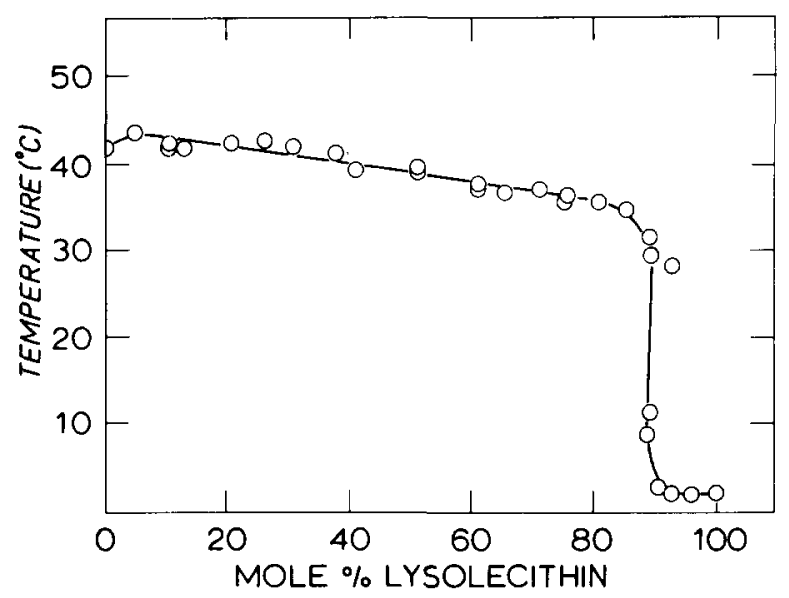

Fig. 3. Effect of lysolecithin on lecithin's transition temperature, taken as the temperature at which the first noticeable divergence from the base line occurred.

for the expected values (fig. 2). We believe that this drop was caused by the gradual formation of a new structure or phase of lecithin and lysolecithin which produced a low, broad transition (fig. 1C). This transition would not be distinguishable from the base line at lower concentrations of lysolecithin; thus, a portion of the enthalpy of transition was not observed. The rise in the observed values at higher lysolecithin concentrations coincided with the appearance of the lysolecithin thermal transition peak.

Increasing the concentration of lysolecithin caused a slow decrease in the transition temperature of the lecithin-lysolecithin mixture (fig. 3). The precipitous drop in the transition temperatures occurred in the region of the intermediate transition and levelled off to the transition temperature of pure lysolecithin.

Freeze etch electron microscopy reveals that with up to $50 \%$ lysolecithin the mixture forms a lamellar phase. However, while up to $30 \%$ lysolecithin normal liposomal structures are formed by the lamellae (fig. $4 \mathrm{a}$ ), at $50 \%$ lysolecithin the lamellae are stacked bilayers (fig. 4b). At higher concentrations of lysolecithin a lamellar phase is still present, but particulate areas are also visible (fig. 4c). It is suggested that these particulate structures may reflect the hexagonal phase, as is found for a mixture of $60 \%$ lysolecithin (from egg lecithin) $+40 \%$ water by Deamer et al. [17]. So above 50 mol\% there is a real phase separation*.

It has to be noted that at lower concentrations lysolecithin (below 20\%) the mixture exhibits an undulated fracture face below the lipid phase transition. This phenomenon is found for several pure lecithins [9]. The homogeneity of the bandpattern indicates that there is no phase separation in the plane of bilayer, but that there is a homogeneous distribution of lysolecithin [9].

*Freeze etching shows that at $25 \mathrm{~mol} \%$ of lysolecithin smooth areas next to areas with bandpatterns are present indicating a phase segregation in the lateral plane of the bilayer. 


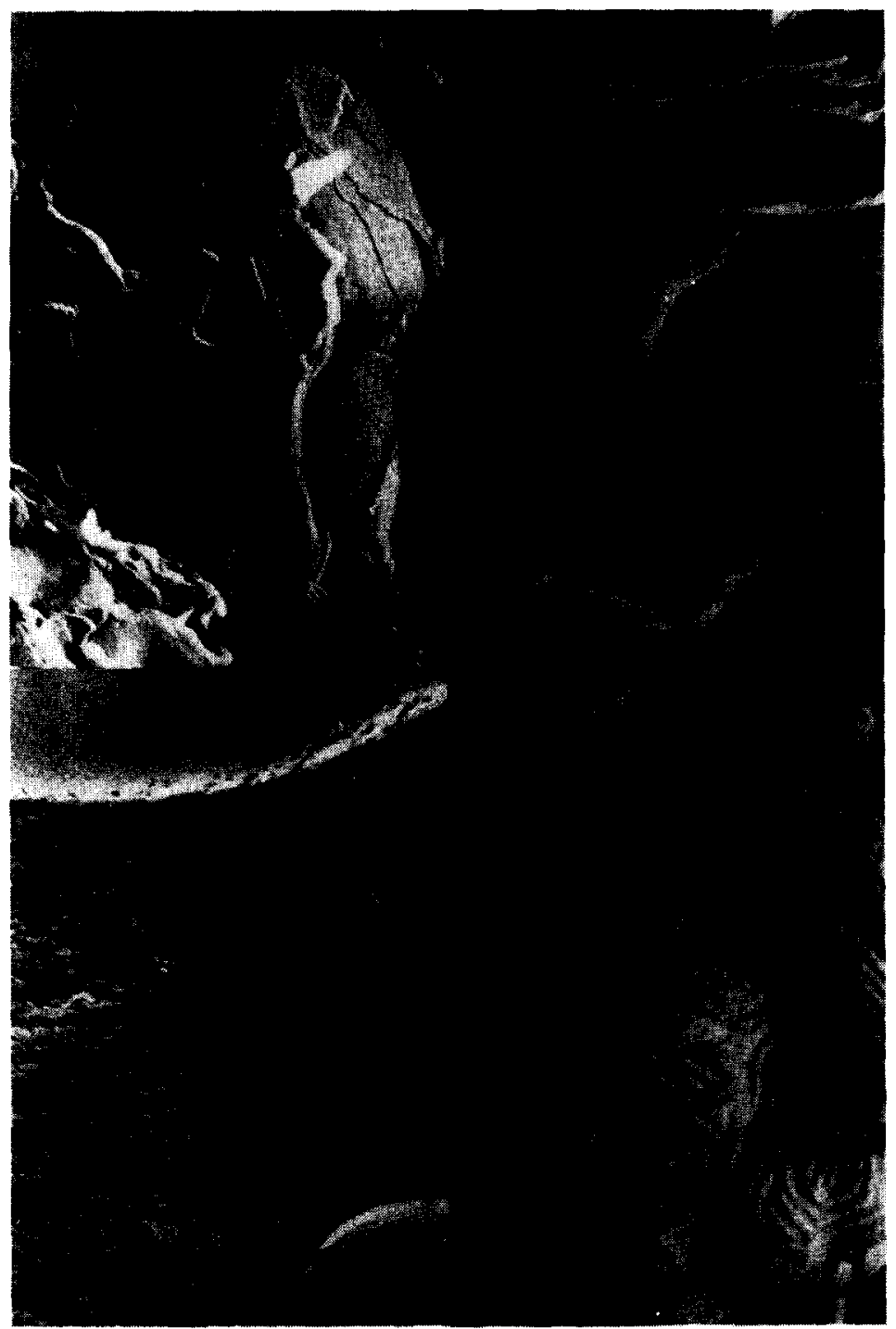

Fig. 4. Freeze etch electron micrographs of mixtures of lecithin and lysolecithin. A. 30\% lysolecithin; B. 50\% lysolecithin; C. $70 \%$ lysolecithin; D. $15 \%$ lysolecithin. The periodicity of the band pattern is about $400 \AA$. All samples were quenched from $23^{\circ} \mathrm{C} . \mathrm{A}, \mathrm{B}$ and $\mathrm{D}$ are fractured at $-196^{\circ} \mathrm{C}$ and $\mathrm{C}$ was fractured and etched during $1 \mathrm{~min}$ at $-100^{\circ} \mathrm{C}$. Magnification about 60,000 .

The effect of cholesterol on the thermal transition of the lysolecithin is shown in figs. 5 and 6 . Initially the enthalpy of transition of lysolecithin increased at low concentrations of cholesterol $(2.5 \%)$ and declined thereafter as concentrations were increased (fig.5). The enthalpy of transition became zero at 50 mole\% of cholesterol. 


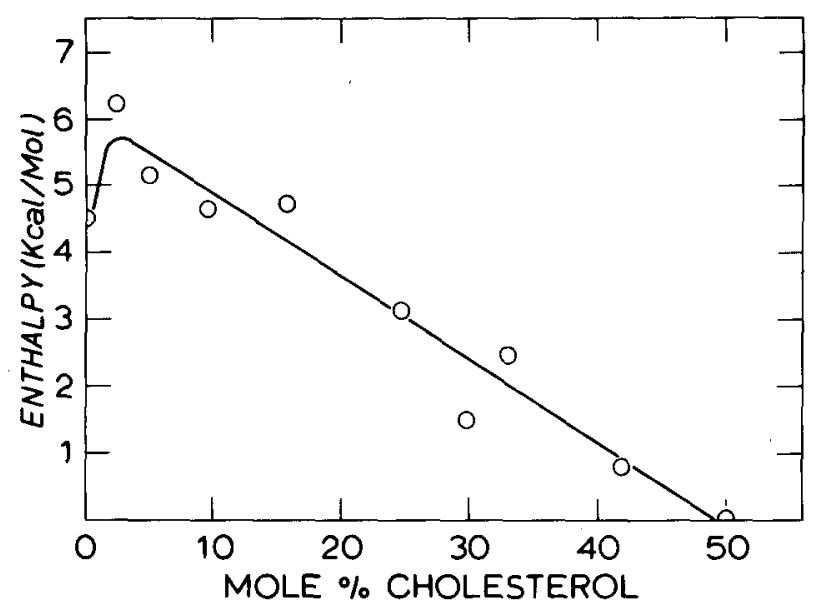

Fig. 5. Effect of cholesterol on the enthalpy of transition of lysolecithin. Stock solutions of cholesterol and lysolecithin were mixed and treated as described in the legend to fig. 1. Enthalpy per mole of phospholipid was calculated as described in fig. 2 .

Cholesterol has been reported to eliminate the transition of lecithin when present at $50 \mathrm{~mole} \%$ [12] or $33 \mathrm{~mole} \%$ [13]. At $50 \mathrm{~mole} \%$ of cholesterol, lecithin provides two acyl chains for interaction with the sterol; at $33 \mathrm{~mole} \%$ there are four chains present for each sterol. Engleman and Rothman [14] proposed a model for lecithincholesterol based on the sterol's interaction with four hydrocarbon chains. However, in our case, interaction of lysolecithin at a ratio of $1: 1$ allowed one hydro-

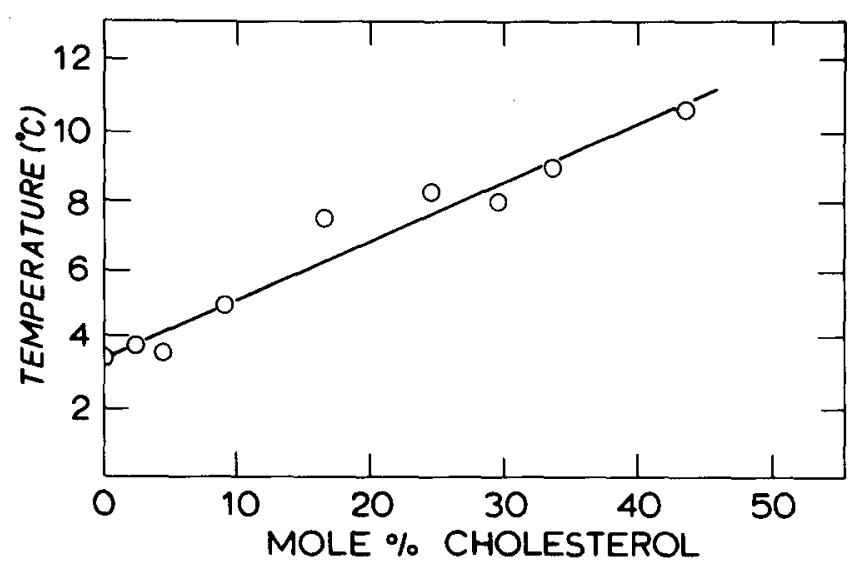

Fig. 6. Effect of cholesterol on the transition temperature of lysolecithin. The temperature of transitions were determined as described in fig. 3 . 
carbon chain for each sterol molecule. Thus, the model proposed for the lecithincholesterol system cannot be extended to the lysolecithin-cholesterol system.

Fig. 6 illustrates another difference between the lecithin-cholesterol and the lysolecithin-cholesterol systems: the transition temperature of lysolecithin increased uniformly with increasing cholesterol concentrations, whereas Phillips et al. [12] observed a linear decrease in the transition temperature of lecithin with increasing cholesterol concentration.

A possible explanation of the behavior of the lysolecithin-cholesterol system may be provided by the statement of Dervichian $[15,16]$ that an equimolar mixture of these compounds exists in a lamellar, liquid crystalline structure. Thus, the cholesterol causes the lysolecithin to adopt a structure similar to that of lecithin. Accompanying this change in structure, the transition temperature of the lysolecithin changes in the direction of that of the diacyl compound.

\section{Acknowledgements}

The authors wish to thank Dr. A. Schuyff and Drs. F. Brants of the Department of Crystal Chemistry of the University of Utrecht for the use of the calorimeter and their assistance with this study. We also wish to thank Jacqueline Mandersloot and Wouter Geurts van Kessel who supplied some of the lipids used.

\section{References}

[1] F.C. Reman, R.A. Demel, J. de Gier, L.L.M. van Deenen, H. Eibl and O. Westphal, Chem. Phys. Lipids 3 (1969) 221

[2] H.U. Weltzien, Biochim. Biophys. Acta 311 (1973) 6

[3] S.A. Ibrahim and R.H.S. Thompson, Biochim. Biophys. Acta 99 (1965) 331

[4] H. van Zutphen and L.L.M. van Deenen, Chem. Phys. Lipids 1 (1967) 389

[5] F.C. Reman, Thesis, University of Utrecht, Utrecht, The Netherlands (1971)

[6] L.L.M. van Deenen and G.H. de Haas, Advan. Lipid Res. 2 (1964) 168

[7] C.H. Fiske and Y. Subbarow, J. Biol. Chem. 66 (1925) 375

[8] P.H.J.T. Ververgaert, P.F. Elbers, A.J. Luitingh and H.J. Van den Berg, Cytobiologie 6 (1972) 86

[9] P.H.J.T. Ververgaert, A.J. Verkleij, P.F. Elbers and L.L.M. van Deenen, Biochim. Biophys. Acta 311 (1973) 320

[10] J.L. Lippert and W.L. Peticolas, Proc. Natl. Acad. Sci. U.S. 68 (1971) 1572

[11] G. Giannoni, F.J. Padden, Jr. and R.F. Roe, Biophys. J. 11 (1971) 1018

[12] M.C. Phillips, R.M. Williams and D. Chapman, Chem. Phys. Lipids 3 (1969) 234

[13] H.J. Hinz and J.M. Sturtevant, J. Biol. Chem. 247 (1972) 6071

[14] D.M. Engleman and J.E. Rothman, J. Biol. Chem. 247 (1972) 3694

[15] D.G. Dervichian, Trans. Faraday Soc. 42B (1946) 180

[16] D.G. Dervichian, Molecular Crystals 2 (1966) 55

[17] D. Deamer, R. Leonard, A. Tardieu and D. Branton, Biochim. Biophys. Acta 219 (1970) 47

[18] W. Luzatti, in: Biological membranes, physical fact and function, ed. by D. Chapman, Academic Press, New York (1968) 71 